\title{
Síndrome de Reacción a drogas con Eosinofilia y Síntomas Sistémicos (DRESS) asociados a Nefritis Túbulo Intersticial Äguda en un adolescente
}

Inés Bibiana Bustamante Gañán a

${ }^{a}$ Médica Especialista en Pediatría, Magíster en Salud Pública. Hospital Infantil Rafael Henao Toro, Manizales, Caldas. Facultad de Ciencias de la Salud, Universidad de Manizales, Caldas, Colombia. ORCID: https://orcid.org/0000-0002-2779-4862, i.bustamante@umanizales.edu.co.

DOI $10.22517 / 25395203.24661$

\section{Resumen}

El síndrome de DRESS (Reacción a drogas con eosinofilia y síntomas sistémicos) es una patología poco frecuente en Pediatría, descrita por primera vez en 1996, por Bocquet. Puede presentarse en un tiempo variable luego de exposición a algunos medicamentos, se caracteriza por fiebre, compromiso cutáneo y de órganos internos. En este caso, se presenta a un paciente de 13 años, con antecedente de uso de Trimetroprim sulfa desde hace 2 meses, con cuadro de 3 días consistente en fiebre y rash cutáneo, sin compromiso de mucosas, con respuesta no favorable al manejo con esteroide, requiriendo Inmunoglobulina IV. Semanas después del inicio de los síntomas y evolución estable presenta insuficiencia renal aguda que requirió terapia de reemplazo renal. Se descartaron otras patologías subyacentes de índole autoinmune. Hubo recuperación de azoados y normalización de los demás paraclínicos el día 40 de la enfermedad. El paciente continúa asintomático, 4 meses después, con tratamiento con esteroide oral, en descenso lento y gradual. Se debe considerar la evaluación permanente de las pruebas de función renal en los pacientes que presenten Síndrome de DRESS, por su asociación con Nefritis intersticial aguda y complicaciones relacionadas.

Palabras clave: Síndrome de DRESS, eosinofilia, insuficiencia renal, nefritis intersticial, pediatría

\section{Drug Reaction with Eosinophilia and Systemic Symptoms (DRESS syndrome) associated with Acute Tubulointerstitial Nephritis in an adolescent}

\begin{abstract}
DRESS syndrome (Drug Reaction with Eosinophilia and Systemic Symptoms) is a rare pathology in Pediatrics, first described in 1996 by Bocquet. It can appear in a variable period of time after exposure to some medications, it is characterized by fever, skin involvement and internal organs. A 13-year-old patient is presented, with a history of use of Trimethoprim sulfa for two months, with a disease of three days of evolution, consisting of fever and skin rash, without mucosal involvement, with an unfavorable response to steroid management, requiring Intravenous inmunoglobulin. Weeks after the onset of symptoms and stable evolution, he presented acute renal failure that required renal replacement therapy. Other underlying autoimmune pathologies were ruled out. There was recovery of renal function test and normalization of the other paraclinical on day 40 of the disease. Patient remains asymptomatic four months later, with oral steroid treatment, in slow and gradual decline. Permanent evaluation of renal function tests should be considered in patients with DRESS syndrome, due to its association with acute tubulointerstitial nephritis and related complications.
\end{abstract}

Key words: DRESS Syndrome; Eosinophilia; Renal insufficiency; Nephritis interstitial; pediatric

\section{Introducción}

El síndrome de DRESS (Reacción a drogas con eosinofilia y síntomas sistémicos) es una patología poco frecuente en Pediatría, con incidencia de 1:1000 a 1:10000, menor que en población adulta, descrita por primera vez en 1996, por Bocquet et al (1-4). Puede presentarse en un tiempo variable luego de la exposición a algunos medicamentos, entre 5 a 105 días post-exposición, y se asocia principalmente al uso de anticonvulsivantes $(50 \%)$ y antibióticos $(30,8 \%)(3,5)$

Secaracterizaporfiebre,linfadenopatías,compromisocutáneoydeórganosinternos,entrelosquesedestacanprincipalmente eosinofilia, presencia de linfocitos atípicos y afectación hepática, pero también pueden cursar con daño renal, pancreático, miocárdico y de otros órganos. Para su diagnóstico se tienen en cuenta los criterios de Bocquet y RegiSCAR (Registro de reacciones adversas severas cutáneas a medicamentos), siendo este último el más utilizado en la población pediátrica (3). 
En el caso clínico reportado, se resalta la afectación renal del paciente, con insuficiencia renal aguda semanas después del inicio de los síntomas, que requirió terapia de reemplazo renal en unidad de cuidados intensivos y la consideración de otras patologías subyacentes asociadas a rápido deterioro de la función renal que fueron descartadas mediante pruebas. La literatura muestra que la principal afectación es a nivel hepático y el compromiso renal es reportado en menor proporción $(2,3)$. La biopsia renal confirmó el diagnóstico de Nefritis Tubulointersticial Aguda y 4 meses después de la presentación del cuadro, el paciente permanece asintomático.

\section{Reporte de caso}

Paciente de 13 años que recibe Trimetroprim sulfa desde hace 2 meses, formulado para el manejo de Acné, sin otros antecedentes patológicos, con cuadro de 3 días de evolución consistente en fiebre y rash cutáneo, sin compromiso de mucosas. Suspende antibiótico, con mejoría parcial, pero 2 días después, hay reaparición de fiebre y aumento de lesiones cutáneas, con rash eritematoso, macular, generalizado (aproximadamente compromiso en $80 \%$ de superficie corporal), pruriginoso, sin lesiones ampollosas. Se descarta Covid19. Es hospitalizado para realización de paraclínicos, entre los que se encuentra elevación de transaminasas, dos veces por encima de lo normal, presencia de eosinofilia mayor a $1500 / \mathrm{mm}^{3}$ y linfocitos atípicos (ver tabla 1 ).

Tabla 1. Evolución de paraclínicos

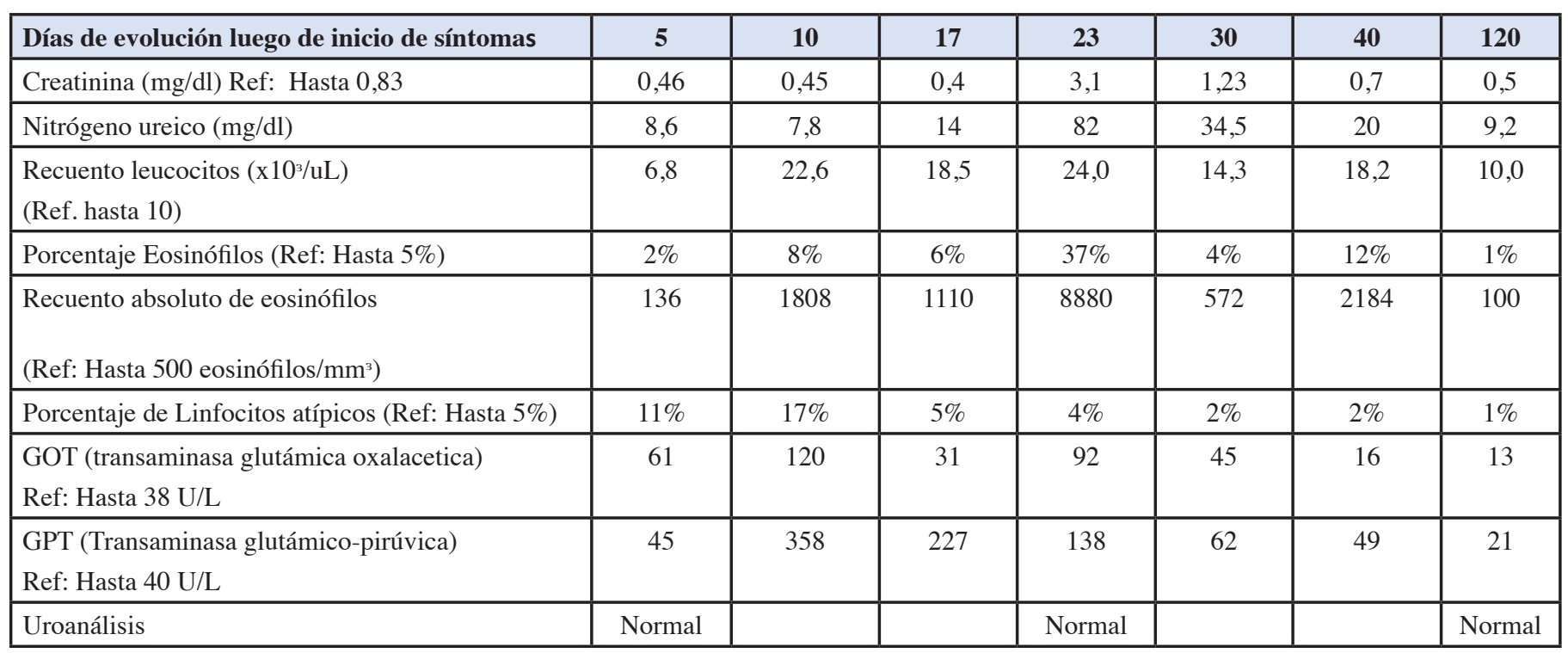

Se sospecha Síndrome de DRESS y se inicia manejo con Metilprednisolona 1 gramo IV (intravenosa) por 3 días, con paso posteriormente a Prednisona oral $1 \mathrm{mg} / \mathrm{kg} / \mathrm{día}$. Pocos días después, luego de haber recibido ciclo de esteroide IV, nuevamente presenta fiebre y exacerbación de lesiones cutáneas, así como persistencia de elevación de transaminasas y eosinofilia, por lo que se ordena manejo con Inmunoglobulina IV $2 \mathrm{~g} / \mathrm{kg}$ por 2 días, con buena respuesta.

Hay evolución clínica estable durante algunos días, pero aproximadamente 23 días después del inicio de la sintomatología y al haber iniciado el descenso de Prednisona, hay reaparición de fiebre, persistencia de lesiones cutáneas, en menor extensión y elevación significativa de azoados, con falla renal aguda que requirió traslado a cuidados intensivos pediátricos para hemodiálisis. Se indica segundo ciclo de Metilprednisolona, 1 gramo IV diario por 3 días. Por rápida progresión de su falla renal, se adiciona otro inmunosupresor, Ciclofosfamida en dosis de $750 \mathrm{mg} / \mathrm{m}^{2}$ (con ajuste a función renal) y amplían estudios para descartar patologías subyacentes de tipo autoinmune. El paciente tuvo elevación de azoados más de 1,5 veces del valor basal, lesión renal aguda KDIGO 3 y depuración de creatinina $36 \mathrm{ml} / \mathrm{min} / 1,73$ $\mathrm{m}^{2}$ ). Con manejo descrito, hubo recuperación de azoados y normalización de los demás paraclínicos el día 40 del cuadro y se mantienen así 4 meses después de la presentación del cuadro clínico. (Gráfico 1) 


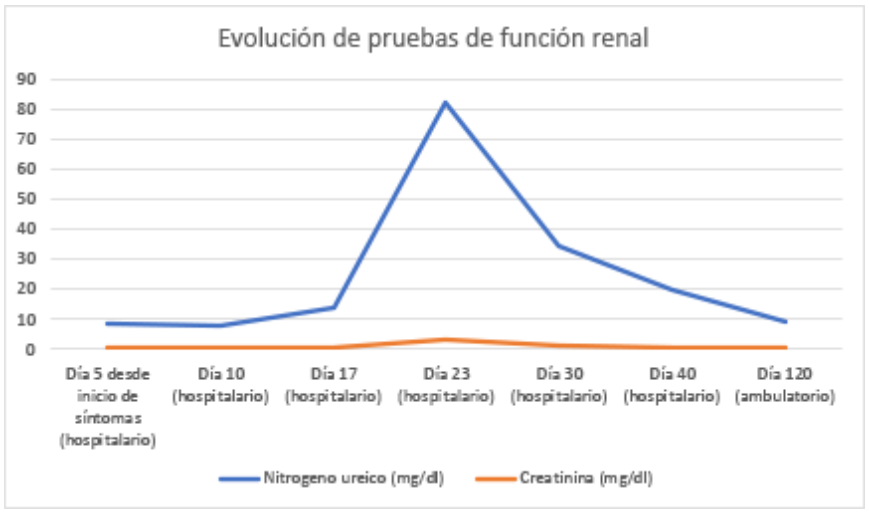

Gráfico 1. Evolución de las pruebas de función renal

El uroanálisis no mostró datos de alarma en ningún momento de la evolución de la enfermedad, no se evidenció hematuria, ni proteinuria ni alteración en sedimento. Se descartó Lupus eritematoso sistémico, tras haberlo considerado por su hipocomplementemia (C4 en $8,5 \mathrm{mg} / \mathrm{dl}$, disminuido y $\mathrm{C} 3$ en $97,2 \mathrm{mg} / \mathrm{dl}$, normal) y deterioro renal descrito. Se descartó Síndrome antifosfolípidos, tuvo estudios alterados en esta fase de su enfermedad: Anticardiolipina Ig M positiva, Anti B2 glicoproteína Ig M positiva y Antifosfolípidos Ig $\mathrm{G}$ y $\mathrm{M}$ positivos, pero al realizar controles posteriores, todos se encontraron en límites normales. Los demás estudios fueron negativos (Anti-DNA negativo; pANCA y cANCA negativos; ANA negativo; ENAS: antiRNP, antiLA, antiSm, antiRo negativos, VDRL y Coombs directo negativos). Se consideró posible relación de este hallazgo con la administración de Inmunoglobulina IV, que puede ser un efecto transitorio, con resolución varias semanas después, como ocurrió en el paciente. Se realizó ecocardiograma en límites normales. Cuatro meses después del inicio del cuadro, el paciente se encuentra asintomático, con tratamiento con Prednisona oral con descenso gradual y lento, según lo recomendado en la literatura.

En los gráficos 2 y 3 se muestra el comportamiento de azoados en relación con transaminasas y hemograma durante la evolución del paciente en los 4 meses iniciales de su atención:
Relación entre comportamiento de Nitrogeno ureico y transaminasas

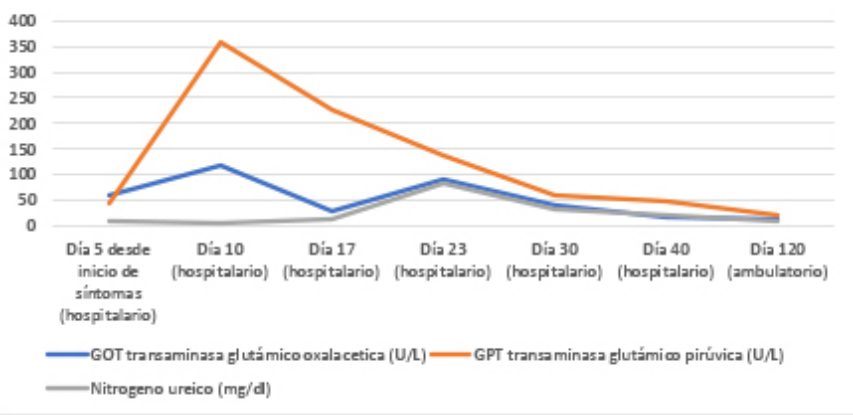

Gráfico 2. Relación entre el comportamiento del Nitrógeno ureico y las transaminasas

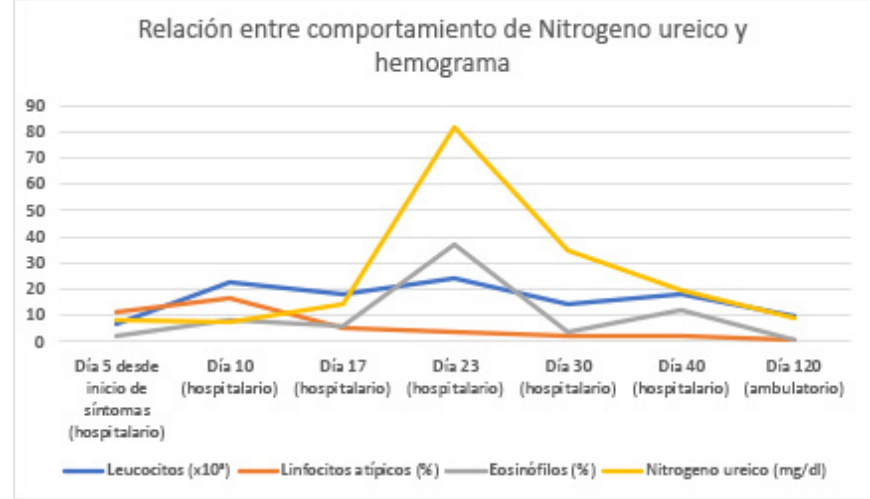

Gráfico 3. Relación entre comportamiento de Nitrógeno ureico y hemograma

El reporte de la biopsia renal Nefritis túbulo intersticial aguda severa, cambios histológicos compatibles con hipersensibilidad medicamentosa. Presencia de Ig A en la inmunofluorescencia sugestivo de Nefropatía por Ig A de fondo con proliferación mesangial mínima, sin proliferación endocapilar, esclerosis segmentaria, sin presencia de proliferación extracapilar, sin cambios que sugieran podocitopatía sobreimpuesta.

\section{Discusión}

El síndrome de DRESS es una reacción sistémica multiorgánica, caracterizada por una triada clínica (rash, fiebre y compromiso sistémico) acompañada de alteraciones en el hemograma como eosinofilia y presencia de linfocitos atípicos y disfunción hepática y renal, como las principales afectaciones mencionadas. El promedio de tiempo de presentación de los síntomas es variable luego de la exposición al medicamento, oscilando entre 3 a 8 semanas o hasta 100 días según otros autores (1-4,6-10).

La incidencia en población pediátrica no está bien establecida, pero se estima que la entidad se presenta entre 1:1000 a 1:10000 exposiciones a medicamentos, 
siendo los anticonvulsivantes (50\%) y antibióticos $(30,8 \%)$ las causas más comunes, con reportes de tasas de mortalidad hasta del $10 \%(3,5,9,11)$.

En la literatura se mencionan enfermedades similares desde hace varias décadas, pero fue en 1996 que Bocquet et al. acuñó el término DRESS para la descripción de reacciones de hipersensibilidad a medicamentos y definió los siguientes criterios para su diagnóstico: erupción cutánea asociada a drogas; fiebre, adenopatía mayor de $2 \mathrm{~cm}$, hepatitis con aumento de transaminasas (más de 2 veces lo normal), nefritis intersticial o miocarditis y alteraciones hematológicas como eosinofilia mayor a $1500 / \mathrm{mm}^{3}$ y linfocitos atípicos. Posteriormente, se desarrolló en Europa, en el año 2007, el sistema de puntuación RegisCAR (Registry of Severe Cutaneous Adverse Reaction) que clasifica los casos como "No es caso", "Posible", "Probable" y "Definitivo", teniendo en cuenta elementos clínicos y de laboratorio. Este puntaje fue publicado originalmente por Kardaun et al, con una puntuación inicial (criterios de inclusión), seguida de la confirmación (criterios de validación). (3,11-14).

El caso presentado tuvo un puntaje mayor de 5, según RegisCAR, siendo catalogado como caso definitivo, con presencia de fiebre, compromiso de piel sugerente de la noxa de más del $50 \%$ en extensión, eosinofilia (recuento absoluto mayor de 1500), linfocitos atípicos y afectación hepática (aumento de transaminasas por encima de 2 veces lo normal) y renal (elevación de azoados más de 1,5 veces del valor basal, lesión renal aguda KDIGO 3 y depuración de creatinina $36 \mathrm{ml} / \mathrm{min} / 1,73 \mathrm{~m}^{2}$ )

El comportamiento del paciente muestra datos típicos de la enfermedad, con el compromiso ya mencionado en piel y hemograma (eosinofilia y linfocitos atípicos), pero en este caso además se resalta el daño renal por lo que el paciente requirió terapia renal (hemodiálisis), que en la literatura poco se menciona. La afectación de otros órganos internos, como el hígado se reporta en un 50$80 \%$ de los pacientes, el riñón entre 11-28\% y menos frecuentes el pulmón en 2-5\%, sistema gastrointestinal en $8 \%$ y corazón en $4 \%(3,4)$.

Entre los diagnósticos diferenciales que se asocian al rápido compromiso renal detectado en este paciente figuran patologías autoinmunes, como lupus eritematoso, síndrome antifosfolípidos, neoplasias hematológicas de precursores eosinofílicos, enfermedad de Kawasaki, otras eritrodermias e infecciones bacterianas y virales. Los estudios solicitados en este sentido descartaron estas enfermedades y la evolución clínica y biopsia renal confirman el diagnóstico de Nefritis tubulointersticial aguda severa, compatibles con hipersensibilidad medicamentosa.
La nefritis tubulointersticial aguda (NTI) es un tipo de daño renal, potencialmente reversible, con curso variable, entre manifestaciones subclínicas hasta falla renal, con múltiples etiologías, siendo las más frecuentes las reacciones a medicamentos, en aproximadamente el $70 \%$ a $90 \%$ de los casos, seguido de infecciones y enfermedades autoinmunes. En niños además se reportan mutaciones genéticas. Entre los medicamentos figuran AINEs (antiinflamatorios no esteroideos), antibióticos como beta lactámicos, sulfas y cefalosporinas, anticonvulsivantes e inhibidores de bombas de protones. La NTI es la responsable de 5 a $15 \%$ de las injurias renales agudas en niños y adultos y pueden desarrollar enfermedad renal crónica (15-17).

Finalmente, en cuanto al manejo en este paciente se resalta el uso inicial de esteroide intravenoso (Metilprednisolona 1 g/día, dosis máxima por su peso) por 3 días, con posterior uso de esteroide oral (Prednisona $1 \mathrm{mg} / \mathrm{kg}$ / día), sin respuesta esperada en los siguientes días, dada persistencia de síntomas cutáneos y sistémicos, por lo que requirió aplicación de Inmunoglobulina Intravenosa $(2 \mathrm{~g} / \mathrm{kg})$ y un segundo ciclo de Metilprednisolona en igual dosis. Se continuó dosis de Prednisona $1 \mathrm{mg} / \mathrm{kg} / \mathrm{día}$. La respuesta fue favorable con mejoría de lesiones en piel y con parámetros bioquímicos controlados, pero 23 días después de estar recibiendo su manejo con esteroide oral y habiendo iniciado su descenso de dosis, el paciente presenta la complicación renal descrita que lleva a internación en cuidados intensivos para hemodiálisis. Paralelamente, Nefrología pediátrica indica manejo con Ciclofosfamida, una dosis y se descartan otras etiologías. El paciente tiene respuesta favorable, la cual se mantiene luego de 4 meses de manejo y seguimiento ambulatorio, realizando descenso más gradual de su tratamiento con Prednisona, según lo que sugiere la literatura en estos casos de afectación renal para evitar recaídas (8$10,12,13,16)$.

\section{Conclusiones}

El síndrome de DRESS es una entidad poco frecuente en Pediatría, encontrando cada vez más reportes de casos, con medicamentos de uso común, por lo que el personal médico debe realizar vigilancia y seguimiento estrecho de sus pacientes al momento de formularlos y sospechar la entidad prontamente ante síntomas clásicos de la misma como fiebre, erupción cutánea, adenopatías y cambios en laboratorios básicos que pueden realizarse en niveles primarios de atención como eosinofilia, presencia de linfocitos atípicos, elevación de transaminasas y de azoados, para el inicio de manejo oportuno, que, en primera instancia, es la suspensión inmediata del medicamento sospechoso y el uso de esteroides (oral o intravenoso) y las subsiguientes recomendaciones, según la evolución clínica del paciente, que deben definirse en 
centros de mayor complejidad. Debe tenerse presente que las recomendaciones frente al uso de esteroide oral ambulatorio incluyen un descenso mucho más gradual y lento (semanas o meses).

La afectación renal puede encontrarse en etapas tardías de la enfermedad, semanas después de la presentación inicial de síntomas sistémicos, por lo que se recomienda dentro del seguimiento del paciente, la realización de estas pruebas de función renal, aún meses después del diagnóstico inicial. Además, ante compromiso renal rápido y agresivo, deben contemplarse exámenes diagnósticos que descarten otras patologías subyacentes en el paciente, como enfermedades autoinmunes.

Es fundamental el diagnóstico temprano y el manejo interdisciplinario para obtener resultados favorables en los pacientes y disminuir el riesgo de complicaciones graves y pronósticos desfavorables.

Financiación: No existe ninguna fuente de financiación externa.

Conflicto de intereses: No existe ningún conflicto de intereses

Confidencialidad de los datos y consentimiento informado: La autora declara que ha seguido los protocolos de su centro de trabajo sobre la publicación de datos de pacientes, contando con la aprobación respectiva del Comité de Investigaciones, con el consentimiento informado del acudiente y el asentimiento del paciente.

\section{Referencias}

1. Karakayali B, Yazar AS, Çakir D, Cetemen A, Kariminikoo M, Deliloglu B, et al. Drug Reaction with Eosinophilia and Systemic Symptoms (DRESS) syndrome associated with cefotaxime and clindamycin use in a 6 year-old boy: a case report. Pan Afr Med J [Internet] 2017; 28:1-5. DOI: 10.11604/pamj.2017.28.218.10828.

2. Shiohara T, Mizukawa Y. Drug-induced hypersensitivity syndrome (DiHS)/drug reaction with eosinophilia and systemic symptoms (DRESS): An update in 2019. Allergol Int [Internet] 2019; 68(3):301-8. DOI: 10.1016/j. alit.2019.03.006

3. Mori F, Caffarelli C, Caimmi S, Bottau P, Liotti L, Franceschini F, et al. Drug reaction with eosinophilia and systemic symptoms (DRESS) in children. Acta Biomed 2019; 90(8):66-79. DOI: 10.23750/abm.v90i3-S.8167

4. Criado PR, Criado RFJ, Avancini JM SC. Drug Reaction with Eosinophilia and Systemic Symptoms (DRESS) / Drug-induced
Hypersensitivity Syndrome (DIHS): A review of current concepts. An Bras Dermatol 2012; 87(3). p. 435-49.

5. Metterle L, Hatch L, Seminario-Vidal L. Pediatric drug reaction with eosinophilia and systemic symptoms: A systematic review of the literature. Pediatr Dermatol [Internet] 2020 Jan 5; 37(1):124-9. DOI: 10.1111/pde.14044

6. Watanabe H. Recent Advances in Drug-Induced Hypersensitivity Syndrome/Drug Reaction with Eosinophilia and Systemic Symptoms. J Immunol Res [Internet] 2018; 2018:1-10. DOI: $10.1155 / 2018 / 5163129$

7. Nguyen M, Chen Y, Tartar D. Characteristics of patients hospitalized for drug reaction with eosinophilia and systemic symptoms (DRESS) at a level 1 trauma center. J Invest Dermatol [Internet] 2019 Sep; 139(9):B13. Available from: https://linkinghub.elsevier.com/retrieve/pii/ S0022202X19316926

8. Del Olmo-Gil E, Galindo-López MP, MayaGutiérrez A, Maya-Granados N, BarragánMartínez JA, Alexanderson-Rosas G, et al. Síndrome de DRESS. Presentación de 2 casos y revisión de la literatura [Internet] Vol. 77, Rev Med Hosp Gen Méx. 2014. Available from: www. elsevier.es/

9. Silva-Feistner M, Ortiz E, Rojas-Lechuga MJ, Muñoz D. Síndrome de sensibilidad a fármacos con eosinofilia y síntomas sistémicos en pediatría. Caso clínico. Rev Chil Pediatr. 2017; 88(1):1648. DOI: $10.1016 /$ j.rchipe.2016.05.010

10. Cabañas R, Ramírez E, Sendagorta E, Alamar R, Barranco R, Blanca-López N, et al. Spanish guidelines for diagnosis, management, treatment, and prevention of dress syndrome. J Investig Allergol Clin Immunol. 2020; 30(4):229-53.

11. Tomani M, Caridi C, Charlot C. Complicated Drug Reaction with Eosinophilia and Systemic Symptoms ( DRESS ) Syndrome History in a 14-Year-Old. Am J Case Rep 2021; 22: 1-7. DOI: 10.12659/AJCR.927951

12. Shanoj K, Joseph S, Shenoy P. Drug reaction with eosinophilia and systemic symptoms syndrome. Indian J Rheumatol. 2019; S59-66. DOI: $10.4103 / 0973-3698.272151$

13. Fernández-Reyes MM, Ramírez-Rosales A, Guerrero-Izaguirre I, López-Romo A. DRESS syndrome. Dermatologia Rev Mex. 2018; 62(6):522-8.

14. Kardaun SH, Mockenhaupt M, Roujeau JC. Comments on: DRESS syndrome. J Am Acad Dermatol [Internet] 2014; 71(5):1000-1000.e2. 
DOI: 10.1016/j.jaad.2013.11.053

15. Nieto-Ríos JF, Ruiz-Velásquez LM, Álvarez L, Serna-Higuita LM. Nefritis tubulointersticial aguda. Revisión bibliográfica. Iatreia. 2020; 33(2):155-66. DOI: 10.17533/udea.iatreia.45

16. Ruebner RL, Fadrowski JJ. Tubulointerstitial Nephritis. Pediatr Clin North Am [Internet] 2019; 66(1):111-9. DOI: 10.1016/j.pcl.2018.08.009

17. Higashi Y, Baba N, Yoshimine H, Sakaguchi I, Kanekura T. Late-onset interstitial nephritis in a patient with drug-induced hypersensitivity syndrome/drug reaction with eosinophilia and systemic symptoms. J Dermatol. 2020;47(2):1747. DOI: $10.1111 / 1346-8138.15175$ 